\title{
Physical Ageing of the Contact Line on Colloidal Particles at Liquid Interfaces
}

\section{Citation}

Kaz, David M., Ryan McGorty, Madhav Mani, Michael P. Brenner, and Vinothan N. Manoharan. 2012. Physical ageing of the contact line on colloidal particles at liquid interfaces. Nature Materials 11(2): 138-142.

\section{Published Version}

doi:10.1038/nmat3190

\section{Permanent link}

http://nrs.harvard.edu/urn-3:HUL.InstRepos:9464203

\section{Terms of Use}

This article was downloaded from Harvard University's DASH repository, and is made available under the terms and conditions applicable to Other Posted Material, as set forth at http:// nrs.harvard.edu/urn-3:HUL.InstRepos:dash.current.terms-of-use\#LAA

\section{Share Your Story}

The Harvard community has made this article openly available.

Please share how this access benefits you. Submit a story.

\section{Accessibility}




\title{
Physical aging of the contact line on colloidal particles at liquid interfaces
}

\author{
David M. Kaz ${ }^{\dagger, 1}$, Ryan McGorty ${ }^{\dagger, 1}$, Madhav Mani ${ }^{2,3}$, Michael P. Brenner ${ }^{2}$, \\ Vinothan N. Manoharan*,1,2 \\ ${ }^{1}$ Department of Physics, Harvard University, Cambridge MA USA \\ ${ }^{2}$ School of Engineering and Applied Sciences, Harvard University, Cambridge MA \\ USA \\ ${ }^{3}$ Present address: Kavli Institute for Theoretical Physics, Department of Physics, \\ University of California, Santa Barbara CA USA \\ $\dagger$ These authors contributed equally to this work \\ *To whom correspondence should be addressed: vnm@seas.harvard.edu
}

Young's law ${ }^{1}$ predicts that a colloidal sphere in equilibrium with a liquid interface will straddle the two fluids, its height above the interface defined by an equilibrium contact angle $\mathrm{e}^{2}$. This equilibrium analysis has been used to explain why colloids often bind to liquid interfaces, an effect first observed a century ago by Ramsden ${ }^{3}$ and Pickering ${ }^{4}$ and later exploited in a wide range of material processes, including emulsification ${ }^{5}$, water purification ${ }^{6}$, mineral recover $y^{7}$, encapsulation ${ }^{8}$, and the making of nanostructured materials ${ }^{910}$. But little is known about the dynamics of binding. Here we show that the adsorption of polystyrene microspheres to a water-oil interface is characterized by a sudden breach and an unexpectedly slow relaxation. Particles do not reach equilibrium even after 100 seconds, and the relaxation appears logarithmic in time, suggesting that complete equilibration may take months. Surprisingly, viscous dissipation appears to play little role. Instead, the observed dynamics, which bear strong resemblance to aging in glassy systems, agree well with a model describing activated hopping of the contact line over nanoscale surface heterogeneities. These results may provide clues to longstanding questions on colloidal interactions at an interface ${ }^{11 / 12}$.

This is the accepted version of the following article:

David M. Kaz, Ryan McGorty, Madhav Mani, Michael P. Brenner, and Vinothan N. Manoharan, "Physical ageing of the contact line on colloidal particles at liquid interfaces." Nature Materials 11 (2): 138-142, 2012. doi: 10.1038/nmat3190

The final, published version may be found on the Nature Materials website.

Supplementary information is also available. 
Kaz, McGorty, Mani, Brenner, Manoharan, Nature Materials 11 (2): 138, 2012

Nearly all models of colloidal interactions and structure at interfaces assume that the particles have reached an equilibrium contact angle with the interface, as determined by Young's law (Fig. 11). The motivation for this assumption is the large reduction of surface energy on binding, which for a micrometer-scale particle can be millions of times the thermal energy $k T$. Thus there is a strong driving force toward equilibrium. But the rate of equilibration depends also on how quickly the two fluids can move along the surface of the solid. This dynamic wetting process has been studied in many macroscopic experiments such as droplet spreading 13 and film dewetting $\frac{14}{4}$, but it remains largely unexplored in colloidal systems, which differ markedly from typical macroscopic systems in geometry and surface properties. On a spherical colloidal particle, the length of the contact line is orders of magnitude smaller than the capillary length, and, due to the high curvature of the particle, a micrometer-scale displacement of the contact line can lead to a radianscale increase in the contact angle. Furthermore, because colloidal particles must be functionalized with charges or other groups to prevent aggregation, the surfaces of colloidal particles differ inherently from those of materials that cannot be dispersed. A few atomic force microscopy experiments have studied how colloidal particles breach an interface $\frac{15] 16}{16}$, but these experiments cannot measure the relaxation to equilibrium, which is the focus of our study.

We use an optical technique, digital holographic microscopy $\frac{17}{17}$, to record the trajectories of micrometer-scale polystyrene spheres as they approach and breach a flat interface between an oil (decane) and a water phase containing glycerol and salt $(\mathrm{NaCl})$, as shown in Fig. 1 1 a. We are able to measure the three-dimensional (3D) position of individual colloidal spheres with $2 \mathrm{~nm}$ precision and sub-millisecond time resolution over a total distance of several micrometers and a total time of up to 100 seconds. The combination of high precision and large dynamic range in both space and time allows us to capture the fine and gross details of the particle trajectory, including its approach to the interface, the moment it breaches, and the relaxation that follows. As we shall show, the wetting dynamics are surprisingly slow. Our analysis shows that systems containing particles adsorbed at interfaces may be out-of-equilibrium on experimental time scales, a result that calls into question the validity of models based on Young's law.

In our experiments, we bring each sphere to the interface and initiate adsorption using weak radiation pressure from a focused laser (Fig. 1a). The total optical force on the particle is less than $1 \mathrm{pN}$, which is too weak to bend the interface more than a few tenths of a nanometer ${ }^{18}$. We find that at sufficiently high salt concentrations, the particle moves upward, begins to plateau, and then sharply transitions into a secondary rise (Fig. 1p). Because the formation of a three-phase 
Kaz, McGorty, Mani, Brenner, Manoharan, Nature Materials 11 (2): 138, 2012
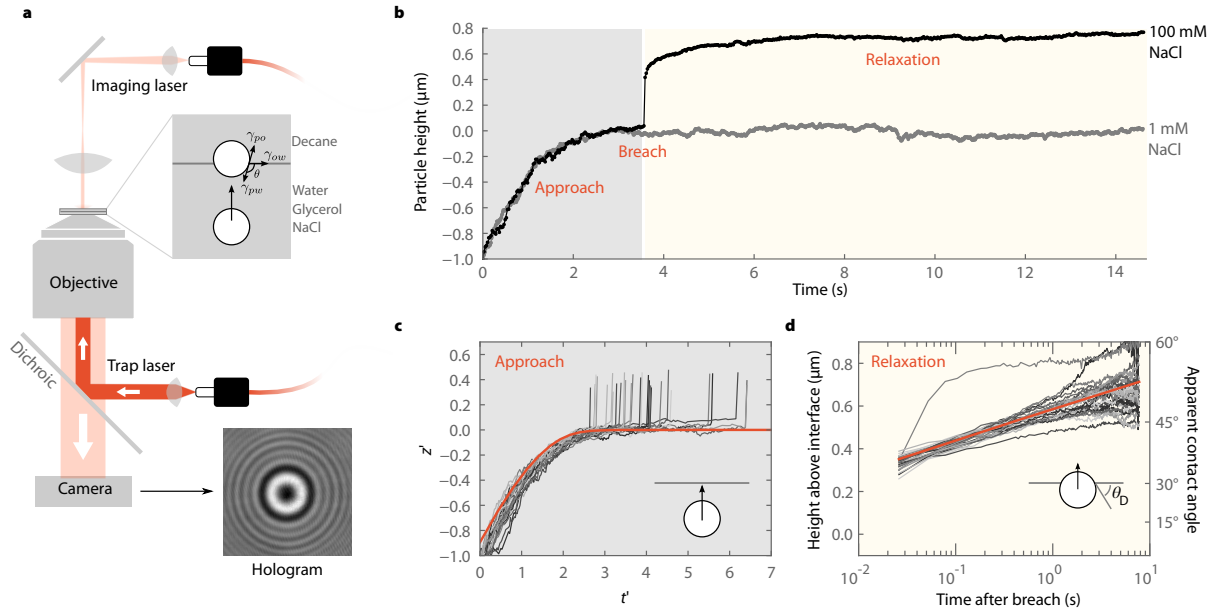

Figure 1: Holographic microscopy reveals dynamics of particles approaching and breaching an interface. (a) Laser light gently pushes a colloidal sphere upward to a water-oil interface, while a second laser illuminates the sphere from above, generating holograms that capture the position of the particle in three dimensions. To obtain high spatial precision $(2 \mathrm{~nm})$ we index-match the two phases, thus eliminating reflections that complicate the analysis of the holograms. (b) Typical trajectories for $1.9-\mu \mathrm{m}$-diameter polystyrene spheres at low and high salt concentrations. We interpret the sharp transition as the point when the particle breaches the interface. (c) The approach to the interface is governed by a balance of drag and radiation pressure. The trajectories for different particles collapse when rescaled by $t^{\prime}=t F_{p} /\left(6 \pi \eta R^{2}\right)$, where $z^{\prime}=\left(z-z_{\text {int }}\right) / R, z_{\text {int }}$ is the position of the interface, $F_{p}$ the force due to radiation pressure, and $\eta$ the viscosity. We include the first point of the discontinuity in each trajectory to show how the breach times are distributed. The red line is the theoretical trajectory. (d) A semi-log plot of position versus time after breach shows that the relaxation is approximately logarithmic. Each grey line is a trajectory for a different particle. The red line shows a logarithmic fit to the average of the data. 
Kaz, McGorty, Mani, Brenner, Manoharan, Nature Materials 11 (2): 138, 2012

contact line cannot be a continuous process, we interpret the discontinuity as the point where the sphere first breaches the interface and interfacial tension starts to drive its motion. This interpretation is supported by several observations. First, after the discontinuity, we cannot pull the particle down with our optical trap. Second, at low salt concentrations, where electrostatics should prevent the particle from breaching 19 , there is no discontinuity, and the particle remains at a plateau (Fig. 1b). Third, as shown in Fig. 1F, the trajectories before the discontinuity agree well with an equation of motion based on the balance of radiation pressure and drag near a planar boundary ${ }^{20}(\mathrm{SI})$.

What happens after the breach is unexpected. The particle trajectories appear logarithmic in time, and even after tens of seconds the system is far from equilibrium: the apparent contact angle is less than $75^{\circ}$, whereas the reported equilibrium value, as measured through a different adsorption method that uses a spreading solvent ${ }^{21}$, is approximately $110^{\circ}$. The motion of the particle is surprisingly slow even in the earliest stages of relaxation: the velocity, roughly $10^{-3} \mathrm{~m} / \mathrm{s}$, and the capillary number $C a=\eta v / \gamma \approx 10^{-4}$ (where $\gamma$ is the interfacial tension) are a thousand times smaller than expected from simple hydrodynamic arguments.

These dynamics, which are qualitatively similar to those of physical aging in other non-equilibrium systems 22 , are difficult to explain with models of capillarity or hydrodynamics, as discussed in detail in the SI. We rule out capillary waves generated by the particle as a possible mechanism for the slow relaxation, as capillary waves are damped on a timescale much shorter than that of particle motion, so the interface quickly equilibrates. We also rule out capillary interactions between neighboring particles, as these are negligible at the low area fractions - at most $10^{-4}$ - of our experiments. A hydrodynamic model accounting for the enhanced viscous dissipation near the moving contact line ${ }^{23}$ does not recover a logarithmic trajectory and grossly over-predicts the particle velocity. Moreover, according to this model, the particle velocity should increase until the contact angle reaches about $60^{\circ}$, in direct conflict with our observations.

The analogy to aging suggests a model that accounts for more local, microscopic processes. One such process is the thermally activated "hopping" of the contact line over surface heterogeneities or defects" ${ }^{24}$. Models of this "molecular dissipation" mechanism use an Arrhenius equation of the form $V=V_{0} \exp (-U / k T+$ $F A / 2 k T)$. This equates the velocity of the contact line, $V$, with a molecular velocity scale, $V_{0}$, times a Boltzmann factor representing the probability that the contact line hops over a defect with activation energy $U$. The rate of hopping over a defect of area $A$ is altered by, and biased in the direction of, the force per unit length acting on the contact line, $F$. The contact line might also hop backward, opposite 
Kaz, McGorty, Mani, Brenner, Manoharan, Nature Materials 11 (2): 138, 2012

the direction of the force, but the probability of backward hops is negligible unless the system is near equilibrium (SI).

In essence, the above Arrhenius model treats surface defects as sites where the fluid interface is pinned with an energy $U$. The model does not distinguish between topographical or chemical defects. It shows that increasingly severe defects, which correspond to larger values of $U$, result in smaller contact-line velocities. In this model, $U$ can also be interpreted as the parameter that determines the contactangle hysteresis 25 .

For our system, the contact line velocity with respect to the sphere is given by $V=R d \theta / d t$, and the force $F$ is the sum of the tangential components of the three interfacial tensions (Fig. S2). Accounting for the variation of $F$ with the position of the sphere (SI), we find an equation of motion for the height of the particle

$$
\dot{z}=\nu \sqrt{z(2 R-z)} \exp \left(-\frac{A \gamma_{o w} z}{2 R k T}\right)
$$

where $\dot{z}=d z / d t$ is the centre-of-mass velocity and $\nu=\left(V_{0} / R\right) \exp (-U / k T+$ $\left.\left[1-\cos \theta_{E}\right] A \gamma_{o w} / 2 k T\right)$ is a constant.

The model reproduces a logarithmic trajectory when the contact angle is close to its equilibrium value (SI). This functional form arises because the driving force $F$ decreases linearly with $z ; F$ is largest when the particle first breaches the interface and decreases to zero at the equilibrium contact angle. Physically, the particle slows down because the interfacial force imposed on the particle decreases as it moves up, so that the contact line requires a larger thermal "kick" to hop over surface heterogeneities. Similar models have been used to resolve the contact line motion in other systems ${ }^{2627}$ at low capillary number and for small changes in $\theta$.

With two fitting parameters (SI) we find good agreement between the Arrhenius model and our data (Fig. 2a). The model fits each individual trajectory at timescales ranging from several milliseconds to several seconds. Deviations occur at times shorter than $10 \mathrm{~ms}$, when a different process, perhaps hydrodynamic, determines the behaviour, and at times longer than a few seconds, when instrumental drift begins to dominate the measurement. To extend the measurements to longer times, we recorded holograms of multiple particles binding in the same field of view. Analysis of this data allows us to remove the drift, which appears as a common mode of the particle trajectories. The resulting, drift-corrected trajectories show that the logarithmic scaling holds over more than three decades of time, up to at least 100 seconds (Fig. 20).

A three-dimensional reconstruction of the holographic data shows that the 
Kaz, McGorty, Mani, Brenner, Manoharan, Nature Materials 11 (2): 138, 2012
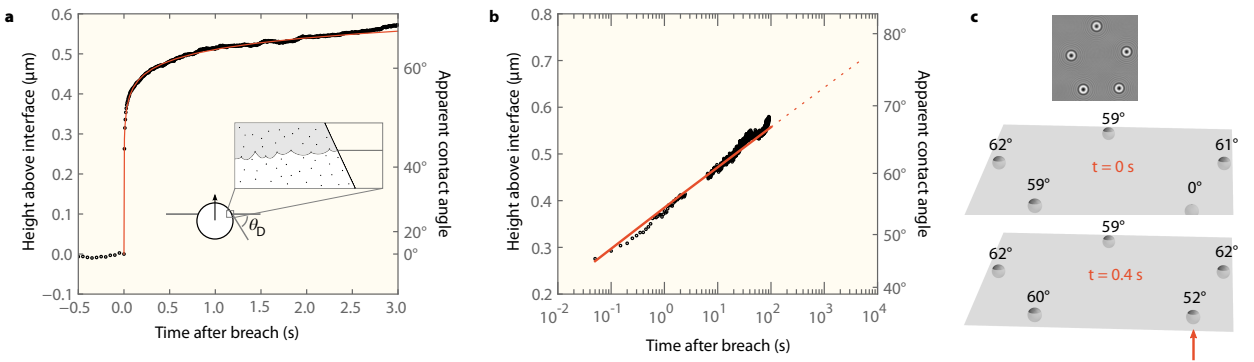

Figure 2: A dynamic wetting model explains the long relaxation and logarithmic dynamics. (a) Relaxation data from the trajectory in Fig. 1b (black) compared to best fit from dynamic wetting model, shown in red. Inset illustrates the premise of the model: contact line pinning at surface defects. (b) Semi-log plot of a particle trajectory with drift subtracted off, showing that the logarithmic scaling holds up to at least $100 \mathrm{~s}$. Best fit to model is shown in red. (c) Analysing a hologram (top) taken just before a particle breaches the interface reveals the plane of the interface and the relative positions of all five particles. Diagrams show renderings of the data before and after the fifth particle breaches the interface. Apparent contact angles are shown above each particle. Arrow indicates the particle tracked in $\mathbf{b}$.

contact angles of different particles can differ by $50^{\circ}$ or more at a given time (Fig. 22), because some particles have breached the interface whereas others remain below (see reconstruction at $t=0$ ). The much smaller discrepancies in contact angle at later times $(t=0.4 \mathrm{~s})$ arise because we forced the particles to breach within a few seconds of one another. In uncontrolled adsorption, the breach times are broadly distributed, and therefore the contact angles can vary widely from particle to particle.

We can estimate the relaxation time of a single particle by extrapolating from the fit in Fig. 2 $\mathrm{b}$. This extrapolation does not account for backward hops of the contact line, which become more probable near equilibrium. Accounting for backward hops in the Arrhenius model shows that the relaxation becomes sublogarithmic near equilibrium (SI); thus our extrapolation should underestimate the relaxation time. With this caveat in mind, we find that the particles should take hours to reach $\theta=90^{\circ}$. To reach the expected equilibrium value ${ }^{\sqrt{21}}$ of $\theta=110^{\circ}$, they may take months - much longer than typical experimental time scales.

Accounting for the effect of the surface heterogeneities on the motion of the contact line permits a mechanistic understanding of the small and decreasing particle velocities. To further investigate these effects we measured trajectories for 
Kaz, McGorty, Mani, Brenner, Manoharan, Nature Materials 11 (2): 138, 2012

several species of polystyrene spheres with different surface functionalities. All particles relax slowly, as seen in Fig. $3 \mathrm{a}$, and the model fits all trajectories well. We find no correlation between average defect size and particle size, but the defect size does vary from one species to another (Fig. 3 3 ), confirming that the relaxation dynamics are due to surface properties and not bulk effects such as swelling with oil. The defect area we extract from our data for sulfate-functionalized spheres is on the order of the area per charged group, but it is unclear whether the defects are the charges themselves or some other features, such as asperities. Future experiments on inorganic solids like silica - a material incompatible with our current optical apparatus - may help resolve this question.

Our results show that nanoscale surface features may dramatically affect the wetting dynamics, and thus the binding dynamics, of colloidal solids at fluid interfaces. The relative effects of hydrodynamic dissipation versus molecular dissipation in macroscopic wetting have been the subject of a decades-long debate 2829 . Though it is generally accepted that both types of dissipation play a role in dynamic wetting, only in cases involving small changes in contact angle has molecular dissipation been clearly shown to dominate. It is therefore surprising that in a system such as ours, where the contact angle changes dramatically in a short time, the molecular perspective emerges as the predictor of wetting dynamics.

The results also call into question whether many systems containing adsorbed colloidal matter are in equilibrium. The assumption of equilibrium underlies the interpretation of essentially all experiments on interfacial assembly of particles, but our measurements show that these systems can be out of equilibrium on experimental time scales. The distance of a particle from its equilibrium position at a given time depends not only on the composition of the system, but also on the method used to attach the particle to the interface. This is evidenced by the more than $30^{\circ}$ difference between the contact angles we observe and those seen for the same type of particles deposited with a spreading solvent ${ }^{21}$.

Non-equilibrium effects may partly explain some of the peculiar features of particle-particle interactions in both planar systems and colloidosomes. For example, observations of particle interactions that vary over time and from particle to particle ${ }^{11}$ are not inconsistent with our observations of the slow creep of the contact line. Also, the wide variation in both the structure of colloidal aggregates at an interface ${ }^{10}$ and the measured interparticle potentials ${ }^{30}$ may be related to differences in how samples are prepared and how they relax to equilibrium. A series of careful experimental studies by Park and coworkers ${ }^{31}[32$ has now established that the lateral interactions between particles can be accurately described by a dipolar electrostatic repulsion, which arises from charges on the aqueous side of the 

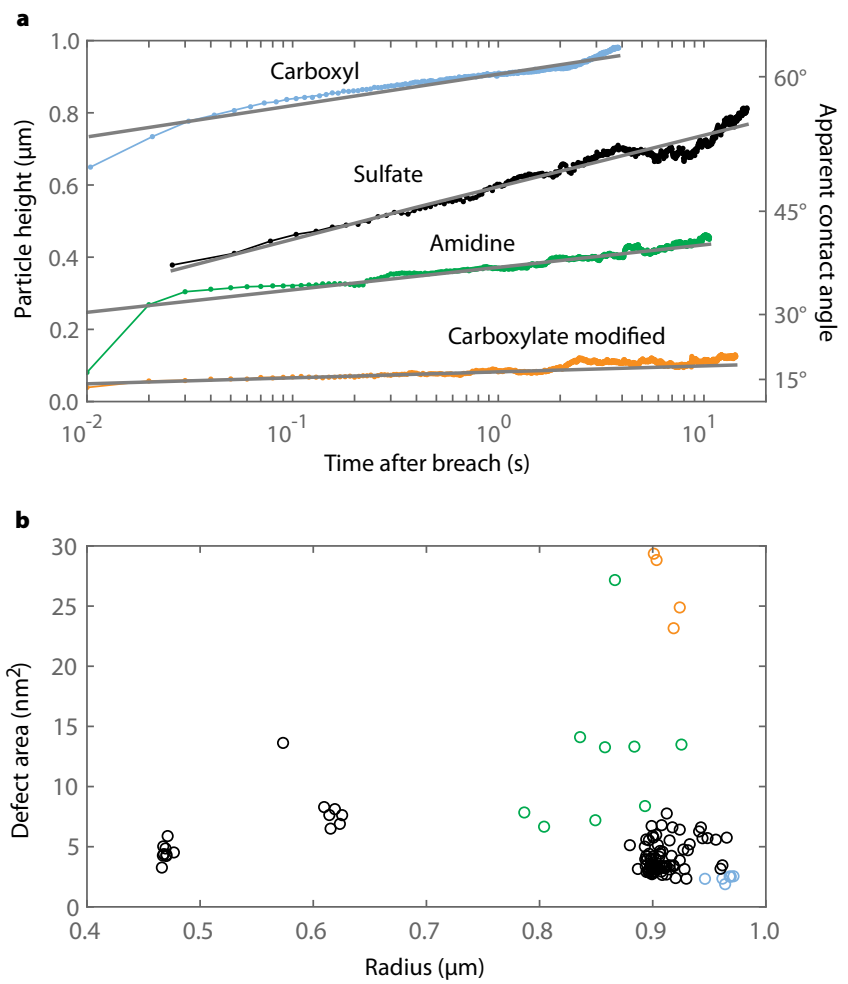

Figure 3: The particle surface controls the rate of relaxation. (a) Particles with different surface functional groups relax at different rates, but all trajectories fit the dynamic wetting model. The four particles whose trajectories are shown in the semi-log plot are all approximately $1.8 \mu \mathrm{m}$ in diameter. Solid lines show best fits to the dynamic wetting model. (b) Defect area, as determined by fitting relaxation trajectories, plotted as a function of particle size and surface functionality. The defect area varies with surface type but is uncorrelated with particle size. The size of each individual particle was determined by fitting the holograms to a Mie-scattering mode 17 . 
Kaz, McGorty, Mani, Brenner, Manoharan, Nature Materials 11 (2): 138, 2012

particle, and a quadrupolar capillary attraction, which is thought to arise from undulations of the contact line due to particle roughness ${ }^{33}$. But it is not clear whether the results of these experiments, which use spreading solvents to deposit the particles on the interface, should apply to systems such as Pickering emulsions ${ }^{5}$ and colloidosomes $\frac{812}{}$, which are prepared using a different method, mechanical agitation.

If the preparation method results in a system that is out of equilibrium, the interactions may differ considerably from those predicted by equilibrium models. For example, we expect the electrostatic repulsion between particles at the interface to vary as the particles relax, since the amount of charged surface exposed to the water phase changes over time. The capillary interactions may vary in a more interesting way. As the contact line moves along the particle surface, its undulations and the accompanying interfacial distortion will fluctuate over time. Because the quadrupolar capillary interaction that arises from this distortion is anisotropic, its direction will also fluctuate. This effect has not, to our knowledge, been considered in studies of particle-particle interactions.

A natural direction for future experiments is to measure both the interactions and the relative heights of particles at the interface. Such experiments may reveal whether some of the most mysterious anomalous interactions ${ }^{12}$ are related to unequal starting times on a surprisingly long road to equilibrium.

\section{Methods}

Optical apparatus. The imaging and trapping laser diodes, as well as all associated optics, were purchased from Thorlabs and assembled next to a Nikon TE2000E inverted microscope. Both laser beams were spatially filtered through singlemode optical fibers to obtain TEM00 profiles. A $100 \times$ oil-immersion objective $(\mathrm{NA}=1.4)$ focused the trapping light (wavelength $830 \mathrm{~nm}$ ) onto a 1-2 $\mu \mathrm{m}$ spot in the image plane of the sample. The counter-propagating imaging laser (wavelength $658 \mathrm{~nm}$ ) was collimated by the microscope condenser $(\mathrm{NA}=0.52)$, yielding a beam waist of roughly $100 \mu \mathrm{m}$ just above the sample. Holograms were captured on a Photon Focus camera (model MV-D1024-160-CL-12) at up to 5000 frames per second.

An immersion oil with roughly the same index of refraction as the sample fluids was used $(\mathrm{n}=1.412)$ instead of the standard oil $(\mathrm{n}=1.51)$. This allowed us to peer deeper into the sample without having to correct for height-dependent spherical aberration. The spherical aberration introduced by using a non-standard immersion oil was insignificant in the central region of the images. This was confirmed by 
Kaz, McGorty, Mani, Brenner, Manoharan, Nature Materials 11 (2): 138, 2012

comparison with aberration-free images, acquired using standard immersion oil.

Sample cells. Custom sample cells (Fig. S1) were made from poly-ether-etherketone (PEEK) and glass coverslips. The sample cells were designed to allow the interface to rest within the working distance of our objective. The PEEK parts were machined in-house and cleaned by sonication in a sequence of HPLC grade methanol, $20 \% \mathrm{v} / \mathrm{v}$ methanol in chloroform, three changes of fresh methanol, and then three changes of fresh deionized water. All glass components, including coverslips and vials, were baked in a pyrolysis oven, sonicated in deionized (DI) water, rinsed, and then blown dry with ultra-pure nitrogen gas. These precautions were taken to minimize contamination by electrolytic or surface-active compounds. The PEEK parts were then adhered to the glass coverslips with UV-cured epoxy (Norland 61) and stored in a vacuum desiccator.

Sample preparation. We use charge-stabilized colloidal suspensions for all of our experiments. Because the charges confer colloidal stability only in a high-dielectric medium, the experiments must start with particles in the aqueous phase rather than the oil phase.

Particles were purchased "surfactant free" from Invitrogen and washed by centrifugation 10 times in DI water before being diluted and stored in sealed vials at $4^{\circ} \mathrm{C}$. Anhydrous decane was purchased from Sigma-Aldrich and purified by filtration through an activated alumina column to remove polar contaminants and a $0.2 \mu \mathrm{m}$ PTFE membrane filter to remove any solids. $\mathrm{NaCl}$ and glycerol were purchased from Sigma-Aldrich. All DI water was produced by a Millipore Milli-Q filtration system, and had a resistivity of $18 \mathrm{M} \Omega-\mathrm{cm}$.

Solutions of $\mathrm{NaCl}$ in water:glycerol $(45: 55 \% \mathrm{w} / \mathrm{w})$ ranging from $0.1 \mathrm{mM}$ to 1.0 $\mathrm{M}$ were made by serial dilution and kept at room temperature. The solutions were filtered through $0.2 \mu \mathrm{m}$ PTFE membranes. The final refractive index of these solutions differed by less than $0.1 \%$ from that of decane. The interfacial tension between the oil and aqueous phase was found to be $\gamma_{o w}=37 \mathrm{mN} / \mathrm{m}$ using a ringtensiometer.

Sample suspensions were prepared by mixing the salt solutions with small volumes of the stock colloids immediately before adding to the sample cells. Cells were partially filled with the aqueous phase and then overfilled with decane. The cells were sealed with glass coverslips that adhered through capillary action. To prevent contamination of the decane, no epoxy was used to seal the top coverslips. After sealing, the samples were allowed to equilibrate for 30 minutes to dissipate small currents associated with temperature gradients and mixing of the two sparingly soluble fluids. During this time, the plane of the interface was kept vertical and 
Kaz, McGorty, Mani, Brenner, Manoharan, Nature Materials 11 (2): 138, 2012

rotated slowly about its normal to keep the slightly buoyant particles from rising prematurely to the interface.

Data acquisition. Experiments were conducted by optically trapping individual particles and then pushing them to a liquid interface. Each particle was first placed 5-10 $\mu \mathrm{m}$ below the interface, then the trap was deactivated, moved downward by $3-$ $5 \mu \mathrm{m}$, and reactivated with the particle above the trap centre. In this configuration, radiation pressure overwhelmed the gradient force so that the force on the particle was upward and nearly constant. This was confirmed by measuring the velocity of a particle in bulk under the influence of the trap and comparing it to the velocity predicted from Stokes drag. The upward force on a 2- $\mu \mathrm{m}$ sphere between 14 and $20 \mu \mathrm{m}$ above the trap centre was about $0.3 \mathrm{pN}$.

Hologram analysis. Holograms were fit to a Mie-scattering model adapted from Lee et al ${ }^{[17}$ to determine the radius, $R$, the refractive index, $n$, and the $x, y$ and $z$ position of the particles. For each trajectory, the first 100 frames were fit by varying all parameters. From these fits, the mean values of $R$ and $n$ were found and used to fit the remainder of the frames for $x, y$, and $z$ alone. The spatial precision was about $2 \mathrm{~nm}$, as estimated from the noise floor of the mean-squared displacement of a freely diffusing particle. Calculations were performed on up to 1,000 64-bit processors on the Odyssey cluster supported by the Harvard FAS Sciences Division Research Computing Group.

Data analysis. Particle trajectories before the breach were fit to the recurrence relation in Equation S4 by the Levenberg-Marquardt method. The same method was used to fit trajectories after breach to a numerical integration of Equation 1 . Measured values of $\gamma_{o w}$ and $T$ were used to extract $A$ from these fits. The value of $\nu$ extracted from the fits was unreliable (see SI).

For the data shown in Fig. 2b, the effects of interfacial drift were removed as follows: The trajectory of the first particle to breach the interface was subtracted from all other trajectories. This removed the common-mode fluctuations, but it also introduced an offset. To compensate for this offset, an extrapolated fit to the first trajectory was added back to all of the others. The location and slope of the interface could then be determined by fitting a plane to the particle positions after breach (Fig. 2c).

\section{References}

1. Young, T. An essay on the cohesion of fluids. Philosophical Transactions of the Royal Society of London 95, 65-87 (1805). 
Kaz, McGorty, Mani, Brenner, Manoharan, Nature Materials 11 (2): 138, 2012

2. Binks, B. Colloidal particles at liquid interfaces. Physical Chemistry Chemical Physics 9, 6298-6299 (2007).

3. Ramsden, W. Separation of solids in the surface-layers of solutions and 'suspensions' (Observations on surface-membranes, bubbles, emulsions, and mechanical coagulation). Proceedings of the Royal Society of London 72, 156164 (1903).

4. Pickering, S. U. Emulsions. Journal of the Chemical Society 91, 2001-2021 (1907).

5. Aveyard, R., Binks, B. P. \& Clint, J. H. Emulsions stabilised solely by colloidal particles. Advances in Colloid and Interface Science 100-102, 503-546 (2003).

6. Nguyen, A., Pugh, R. J. \& Jameson, G. J. Collection and attachment of particles by air bubbles in froth flotation. In Colloidal Particles at Liquid Interfaces (Cambridge University Press, Cambridge, 2006).

7. Bhargava, A., Francis, A. V. \& Biswas, A. K. Interfacial studies related to the recovery of mineral slimes in a water-hydrocarbon liquid-collector system. Journal of Colloid and Interface Science 64, 214-227 (1978).

8. Dinsmore, A. et al. Colloidosomes: Selectively permeable capsules composed of colloidal particles. Science 298, 1006-1009 (2002).

9. Velikov, K. P. \& Velev, O. D. Novel materials derived from particles assembled on liquid surfaces. In Colloidal Particles at Liquid Interfaces (Cambridge University Press, Cambridge, 2006).

10. McGorty, R., Fung, J., Kaz, D. \& Manoharan, V. N. Colloidal self-assembly at an interface. Materials Today 13, 3442 (2010).

11. Park, B. J. et al. Direct measurements of the effects of salt and surfactant on interaction forces between colloidal particles at water-oil interfaces. Langmuir 24, 1686-1694 (2008).

12. Nikolaides, M. et al. Electric-field-induced capillary attraction between likecharged particles at liquid interfaces. Nature 420, 299-301 (2002).

13. Seveno, D. et al. Dynamics of wetting revisited. Langmuir 25, 13034-13044 (2009).

14. Redon, C., Brochard-Wyart, F. \& Rondelez, F. Dynamics of dewetting. Physical Review Letters 66, 715-718 (1991). 
Kaz, McGorty, Mani, Brenner, Manoharan, Nature Materials 11 (2): 138, 2012

15. Dagastine, R. R. \& White, L. R. Forces between a rigid probe particle and a liquid interface II. The general case. Journal of Colloid and Interface Science 247, 310-320 (2002).

16. Ally, J., Kappl, M., Butt, H. \& Amirfazli, A. Detachment force of particles from air-liquid interfaces of films and bubbles. Langmuir 26, 18135-18143 (2010).

17. Lee, S. et al. Characterizing and tracking single colloidal particles with video holographic microscopy. Optics Express 15, 18275-18282 (2007).

18. Park, B. J. \& Furst, E. M. Optical trapping forces for colloids at the oil-water interface. Langmuir 24, 13383-13392 (2008).

19. Mbamala, E. \& von Grunberg, H. Effective interaction of a charged colloidal particle with an air-water interface. Journal of Physics Condensed Matter 14, 4881-4900 (2002).

20. Brenner, H. The slow motion of a sphere through a viscous fluid towards a plane surface. Chemical Engineering Science 16, 242251 (1961).

21. Paunov, V. N. Novel method for determining the three-phase contact angle of colloid particles adsorbed at air-water and oil-water interfaces. Langmuir 19, 7970-7976 (2003).

22. Hodge, I. M. Physical aging in polymer glasses. Science 267, $1945-1947$ (1995).

23. de Gennes, P., Brochard-Wyart, F. \& Quere, D. Capillarity and Wetting Phenomena: Drops, Bubbles, Pearls, Waves (Springer, New York, 2003).

24. Blake, T. D. \& Haynes, J. M. Kinetics of liquid/liquid displacement. Journal of Colloid and Interface Science 30, 421-423 (1969).

25. Rolley, E. \& Guthmann, C. Dynamics and hysteresis of the contact line between liquid hydrogen and cesium substrates. Physical Review Letters 98, 166105 (2007).

26. Prevost, A., Rolley, E. \& Guthmann, C. Thermally activated motion of the contact line of a liquid He meniscus on a Cesium substrate. Physical Review Letters 83, 348-351 (1999).

27. Petrov, J. G., Ralston, J., Schneemilch, M. \& Hayes, R. A. Dynamics of partial wetting and dewetting of an amorphous fluoropolymer by pure liquids. Langmuir 19, 2795-2801 (2003). 
Kaz, McGorty, Mani, Brenner, Manoharan, Nature Materials 11 (2): 138, 2012

28. Blake, T. D. The physics of moving wetting lines. Journal of Colloid and Interface Science 299, 113 (2006).

29. Brochard-Wyart, F. \& Gennes, P. G. D. Dynamics of partial wetting. Advances in Colloid and Interface Science 39, 111 (1992).

30. Oettel, M. \& Dietrich, S. Colloidal interactions at fluid interfaces. Langmuir 24, 1425-1441 (2008).

31. Park, B. J., Vermant, J. \& Furst, E. M. Heterogeneity of the electrostatic repulsion between colloids at the oil-water interface. Soft Matter 6, 5327 (2010).

32. Park, B. J. \& Furst, E. M. Attractive interactions between colloids at the oilwater interface. Soft Matter 7, 7676-7682 (2011).

33. Stamou, D., Duschl, C. \& Johannsmann, D. Long-range attraction between colloidal spheres at the air-water interface: The consequence of an irregular meniscus. Physical Review E 62, 5263-5272 (2000).

Acknowledgements We thank Shankar Ghosh for his help in the work that inspired these experiments; Laura DeLorenzo for her work on sample cell prototypes; Shmuel Rubinstein and Howard Stone for critical discussions; and Jim MacArthur and Stan Cotreau for guidance in construction of the apparatus. This work was supported by the National Science Foundation under CAREER award number CBET-0747625 as well as through the Harvard MRSEC under award number DMR-0820484.

Supplementary Information includes Supplemental Figures and Discussion

Author Contributions R.M. and D.M.K. designed the experimental apparatus, analysed the data, and interpreted the results. M.M. led the development of the model and assisted with interpretation of the data. M.P.B. advised on the model and manuscript. V.N.M. directed the experiments and their interpretation. All authors collaborated on the manuscript.

Competing Interests The authors declare that they have no competing financial interests.

Correspondence Correspondence and requests for materials should be addressed to V.N.M. (email: vnm@seas.harvard.edu). 